\title{
A simple model for the light curve generated by a Shoemaker-Levy 9 impact
}

\author{
Kevin Zahnle \\ NASA Ames Research Center, Moffett Field, California \\ Mordecai-Mark Mac Low \\ Department of Astronomy and Astrophysics, University of Chicago, Chicago, Illinois
}

\begin{abstract}
The impact of a typical Shoemaker-Levy 9 fragment produced three light peaks as seen from Earth. The first peak is related to the entry of the fragment into the Jovian atmosphere. The second peak occurs when the exploding fireball rises above Jupiter's limb into direct view from Earth. The third peak, much the brightest, occurs when the ejecta plume falls back on the atmosphere. By contrast, Galileo, which had a direct view of the impacts, saw two peaks, one at entry, and one at plumefall. Here we present a simple, highly idealized model of a ballistic plume, which we then use to fit the observed light curve of the $\mathrm{R}$ impact as recorded at Mauna Kea and Mount Palomar. From the light curve we find that the nominal $\mathrm{R}$ fragment had diameter $450-500 \mathrm{~m}$ and mass $\sim 2-3 \times 10^{13} \mathrm{~g}$. The uncertainty in the mass is probably about a factor of 3 , with a smaller event more likely than a larger one.
\end{abstract}

\section{The Light Curve}

The general light curve for an impact of a fragment of P/Shoemaker-Levy 9 (SL9), as viewed at infrared wavelengths from Earth, consists of three peaks, the first two faint and brief and the third bright and long-lasting [Graham et al., 1995; Nicholson et al., 1995a]. The three peaks were seen for all the well-observed events (P. Nicholson personal communication, 1995; e.g., see Watanabe et al., [1995], for an excellent recording of the bright $\mathrm{K}$ event at $2.35 \mu \mathrm{m}$ ), but were first reported for the well-observed and well-favored $\mathrm{R}$ event. Figure 1 is a cartoon that illustrates the viewing geometry of a typical SL9 event.

The first peak, or first precursor, preceeded by some 10 seconds the combination entry flash and fireball seen directly by the Galileo spacecraft [Graham et al., 1995; Nicholson et al., 1995a]. As observed from Earth, the first precursor was some 4 orders of magnitude fainter than was the entry flash as seen by Galileo (P. Nicholson personal communication, 1995). At first the fireball was hot enough that it emitted mostly visible light. But as the fireball expanded it cooled. Expansion was effectively adiabatic [Zahnle and Mac Low, 1994a; Chevalier and Sarazin, 1994; Carlson et al., 1995a]. As the temperature dropped the emitted radiation reddened and the plume faded from visible light over some $30-40 \mathrm{~s}$ [Chapman et al., 1995; Martin et al., 1995].

Copyright 1995 by the American Geophysical Union.

Paper number 95JE01620.

$0148-0227 / 95 / 95$ JE-01620\$05.00
The second peak corresponds to the fireball itself rising high enough to be directly visible from Earth [Graham et al., 1995]. That this would happen was predicted by Boslough et al., [1994] and Ahrens et al., [1994]. By this time $(\sim 60 \mathrm{~s})$ the fireball had cooled to $\sim 500-700 \mathrm{~K}$ (Galileo near infrared mapping spectrometer (NIMS), [Carlson et al., 1995a]). Accordingly, the second peak was more prominent at longer wavelengths, as is immediately apparent when the Palomar light curves (3.2 and $4.5 \mu \mathrm{m}$, [Nicholson et al., 1995a]) and the Keck light curve $(2.3 \mu \mathrm{m}$, [Graham et al, 1995$])$ for the $\mathrm{R}$ impact are compared. 'The onset of the second peak was abrupt [Graham et al., 1995]. This can be explained by the fireball having a sharply defined outer edge at early times, which it has in some numerical simulations [e.g., Zahnle and Mac Low, 1994a]. As the fireball continued to expand and cool, it continued to fade.

The fireball expands so enormously that its temperature drops below $50 \mathrm{~K}$. At some point along the cooling trend it becomes inappropriate to call it a "fireball". Rather, the former fireball becomes the vanguard of the ejecta plume. Almost all of its once enormous thermal energy is converted to kinetic energy of expansion. As it expands first silicates condense, later carbonaceous matter, then water (if present), ammonia, and so on. The condensates made the sunlit parts of the plume visible from Earth, to be imaged by the Hubble space telescope (HST) [Hammel et al., 1995].

The third peak corresponds to the plume falling back on the atmosphere and the impact site rotating into view [Zahnle and Mac Low, 1994b; Nicholson et al., 1995a; Graham et al, 1995]. Interestingly, the timing 


\section{Galileo $P$}

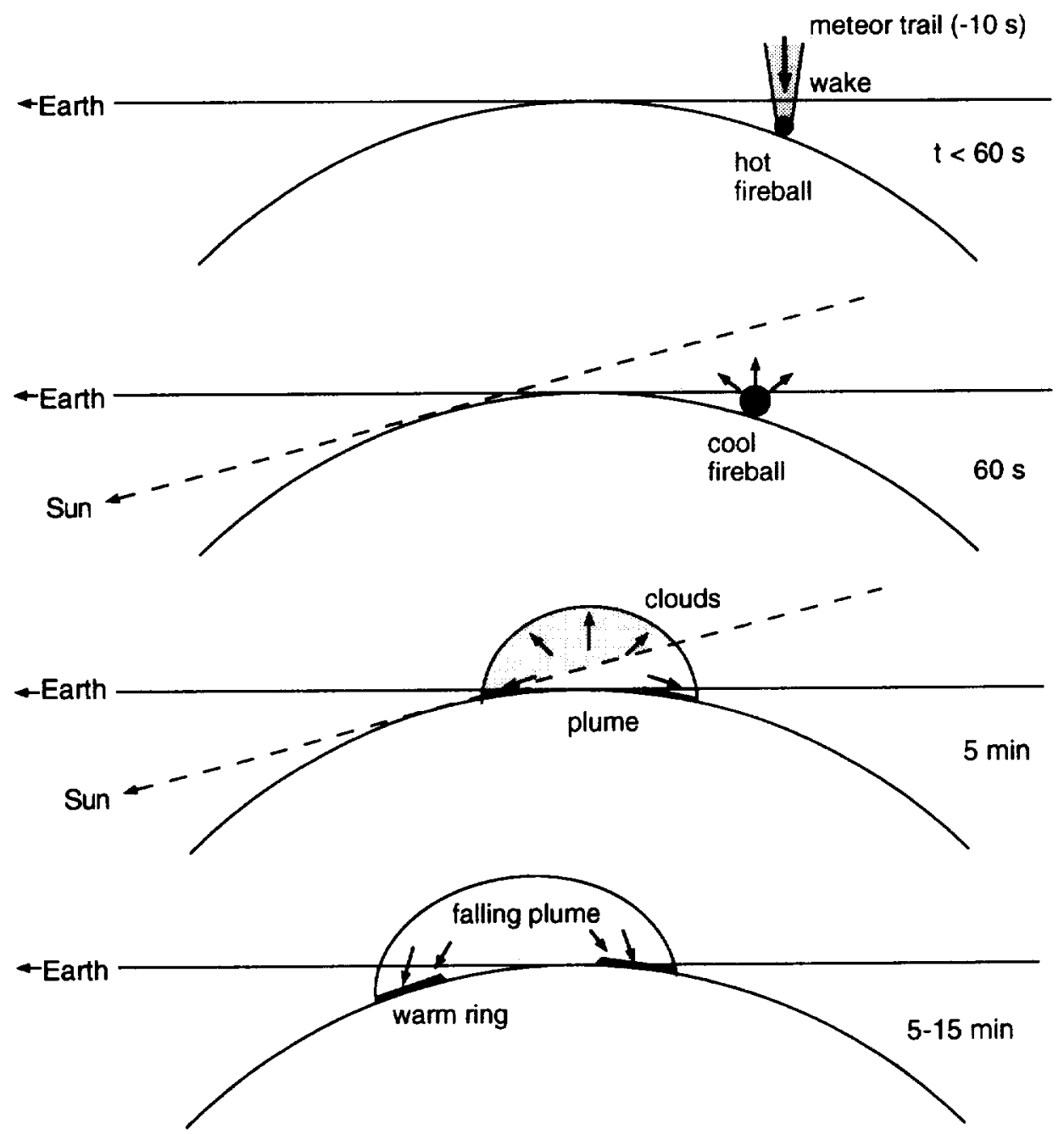

Figure 1. Cartoon illustrating observing geometries for a typical SL9 impact.

of the IR light curve recorded by Galileo for the $R$ and $\mathrm{G}$ events is similar to that observed on Earth [Carlson et al., 1995b]. This implies that something other than Jupiter's rotation delayed the onset of the third peak to 6 min after impact. In contrast to the first two peaks, which account for only a small fraction of the impact energy because the fireball has a small surface area, thermal radiation produced by the plume's reentry into the atmosphere can account for a large fraction of the impact energy, because the plume falls over an enormous area. Most of this paper addresses the thermal radiation produced when the plume falls down.

Still later bumps in the light curve, the so-called bounces, are probably just that, but will not be addressed further in this paper.

\section{Precursors}

Because the impacts occurred on the back side of Jupiter the visibility of the entry flash or fireball on Earth needs explanation. One possibility is that terrestrial observers saw the fireball itself dimly reflected off highaltitude dust, possibly of cometary origin [Hammel et al., 1995]. This no longer seems likely, now that it is well established that the first precursor preceeded the Galileo events by some $10 \mathrm{~s}$. It is likelier that we were seeing direct emission from the high-altitude meteor trail [Graham et al., 1995; Hammel et al., 1995]. Identification with the high altitude meteor trail is strongly favored by the relative timing of the terrestrial and Galileo events [Nicholson et al, 1995a]. The energy is reasonable. Zahnle and Mac Low's [1994a] nominal $1 \mathrm{~km}$ diameter impactor releases about $0.01 \%$ of its energy above $250 \mathrm{~km}$ above the cloud tops, high enough to be directly visible from Earth [Nicholson et al., 1995a]. The emission at infrared wavelengths implies a relatively low temperature. The low temperature argues in favor of the meteor trail, effectively a line charge which, like the fireball, also expands and cools [Boslough et al., 1994; Crawford et al, 1994].

The fireball rises above the limb about a minute after impact. Figure 2 shows fireball light curves calculated 


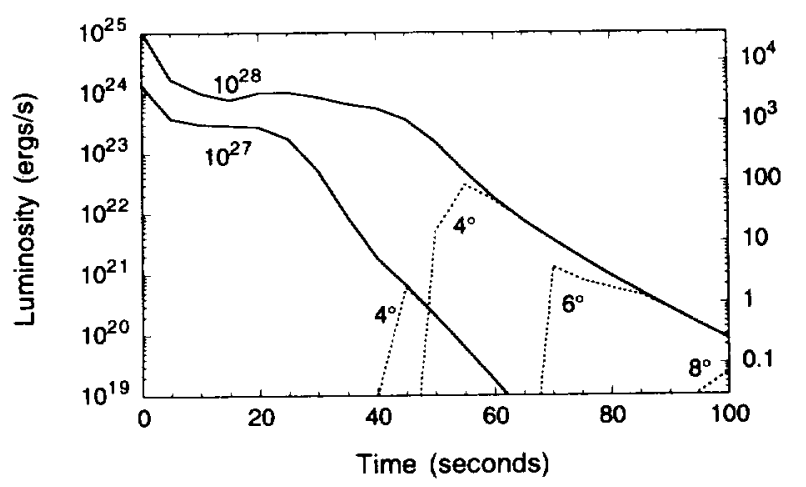

Figure 2. Fireball light curves, calculated numerically after Zahnle and Mac Low [1994a]. Luminosities are extremely sensitive to details in the calculations, and should not be taken too literally. The solid curves loosely approximate Galileo's unobstructed view. The dotted curves represent the view from Earth. They are labeled by the angle past the limb where the impact occurred. The scale labeled "Janskys" is an order of magnitude estimate only.

for $10^{27}$ and $10^{28}$ erg impacts. The solid curves show unobstructed light. They loosely approximate what was seen by Galileo. The dotted curves show the second precursor as viewed from Earth. The labels on the dotted curves give the angle $\theta_{0}$ of the impact site in degrees beyond the limb. The $\mathrm{R}$ impact occurred at about $\theta_{0}=5^{\circ}$.

These calculations are analogous to the numerical light curves predicted by Zahnle and Mac Low [1994a]; our method is described there. Initial conditions for the models shown in Figure 2 are are described in the appendix. The light curves shown here differ from W1 and W2 shown on Figure 12 of Zahnle and Mac Low [1994a] in that they are viewed from the side, they describe smaller events, and the wake (a prescribed initial condition) is hotter. The double peak shown in Figure 12 of Zahnle and Mac Low [1994a] represents something of a conceptual misunderstanding on our part: our calculations stitched together two separate models, one for the entry and another for the explosion, and we did not do this well.

Figure 2 is meant more as an illustrative cartoon than as a serious quantitative model: these calculations were predictions, made before the impact, as our answer to the predictions of fireball visiblity made by Ahrens et al. [1994] and Boslough et al. [1994] We have made no effort to account for the wavelength of the observation, so that the scale labelled "Janskys" is an order of magnitude estimate only. Because radiative cooling is only a tiny fraction of the energy budget of the fireball, calculated luminosities are extremely sensitive to details in the numerical model (especially temperature) and to modest variations in impact parameters. An important limitation is that the only opacity source we considered was the $\mathrm{H}^{-}$ion. The calculated fireball fades too quickly because the $\mathrm{H}^{-}$disappears exponentially with cooling temperatures. In the real plume at temperatures below about $1500 \mathrm{~K}$ the main opacity source would have been molecules or dust, either silicate or carbonaceous. Because it is more opaque, the real fireball would fade more slowly than the calculated fireball [Graham et al., 1995].

Nicholson et al. [1995a] point out that the decay times for the second peak are counterintuitive: the 2.3 micron curve decays much more slowly than the 4.5 micron curve, although the latter was at first much brighter, and further cooling would suggest that fading at $4.5 \mu \mathrm{m}$ would be slower. The resolution may be due to different opacity sources (either molecular or aerosol), as $2.3 \mu \mathrm{m}$ is deep in a methane-carbon monoxide absorption feature, and small aerosols emit better at shorter wavelengths.

\section{The Toy Plume}

As a first model for the infrared light curve, we have devised a simple two-dimensional (2-D) axisymmetric toy model to describe the decline and fall of the plume. The toy plume assumes that the velocity field of the ejecta plume is ballistic and that the mass-velocity distribution in the plume follows a power law that we have calibrated to numerical models. Two additional simplifying assumptions, that the plume is axisymmetric with an opening angle $\theta^{\prime}$, can easily be changed to treat an inclined, asymmetric plume. The ballistic approximation is valid to a factor of order $c_{s}^{2} \ll v^{2}$, where $c_{s}$ is the sound speed and $v$ a typical velocity in the plume, and is in excellent agreement with the results of detailed numerical models.

We divide the ejecta plume by position $(r, \theta, \phi)$ into a vast number of mass elements. Each element is launched on its own unique ballistic trajectory. We tile Jupiter's "surface" by distance and azimuth to produce a kind of dart board centered on the impact site. We then count up where and when the mass elements reenter the atmosphere (i.e., where and when they hit the dart board). The effective radiating temperature of the reentry shock is determined by balancing the energy supplied by infalling ejecta against thermal radiation by opacity sources (dust, soot, molecules, darts, etc.) embedded in - or generated by - the reentry shock. The approach is closely analogous to that used by Melosh et al. [1990] and Zahnle [1990] to model thermal radiation following the $\mathrm{K} / \mathrm{T}$ impact. We account for the viewing geometry of each surface element when integrating the radiated flux as seen on Earth.

\section{Velocity}

The mass-velocity distribution of ejecta from hypervelocity impacts generally obeys a power law. It is convenient to write the power law in cumulative form,

$$
M(>v) \propto v^{-\alpha},
$$

in which the notation $M(>v)$ refers to the cumulative mass ejected at velocities greater than $v$. Examples abound. In a study of ejecta scaling, Housen et al. [1983] cite impact experiments in sand $(\alpha=1.23)$, water $(\alpha=1.66)$, and basalt $(\alpha=2)$. The experimen- 
tally based crater-scaling rules suggested by Schmidt and Housen [1987] are equivalent to flow fields in which $\alpha=1.33$ (nonporous target) or $\alpha=1.8$ (porous target). The power $\alpha$ is constrained by the conservation laws of momentum and energy to be between 1 and 2 [Zel'dovich and Raizer, 1967, p. 834; Housen et al., 1983].

Perhaps more directly relevant to airbursts on Jupiter is an approximate similarity solution for the normal impact of a gas "plate" on a gas half-space [Zel'dovich and Raizer, 1967, pp. 827-833, in particular, equations 8.38-8.42 on p. 828]. Zel'dovich and Raizer define the cumulative mass above an altitude $z$ as $m$, and the cumulative mass above the shock front as $M$. The latter is a monotonically increasing function of time. The upward velocity $u$ increases monotonically with $z$ (in the asymptotic limit, $u$ increases linearly with $z$ ). An analytic solution is obtained for the special case of an ideal gas with $\gamma=1.4$. The cumulative mass $m$ with velocity greater than or equal to $u$ is given by

$$
u \propto M^{-2 / 3}\left((m / M)^{-2 / 3}-3\right) .
$$

In the limit that $m \ll M$, we obtain $u \propto m^{-2 / 3}$. The asymptotic mass-velocity distribution of the ejected gas therefore takes the form $M(>v) \propto v^{-1.5}$. Zel'dovich and Raizer argue that the power $\alpha$ depends on the geometry of the blow-out as well as on $\gamma$, and so we should not necessarily expect $\alpha=1.5$ to apply to threedimensional explosions on Jupiter, nor should we expect the same value of $\alpha$ to apply to impacts of all magnitudes.

Figure 3 shows cumulative velocity distributions at 50 and $100 \mathrm{~s}$ calculated from two hydrodynamic simulations with $\gamma=1.4$. (see appendix for numerical details). The simulations are similar to those published elsewhere by Zahnle and Mac Low [1994a]; cumulative velocity distributions are presented in their Figure 8). Figure 3 was constructed by adding up all the mass on the grid with velocity greater than $v$. We ignore the direction of motion, although most motion is outwards, as one

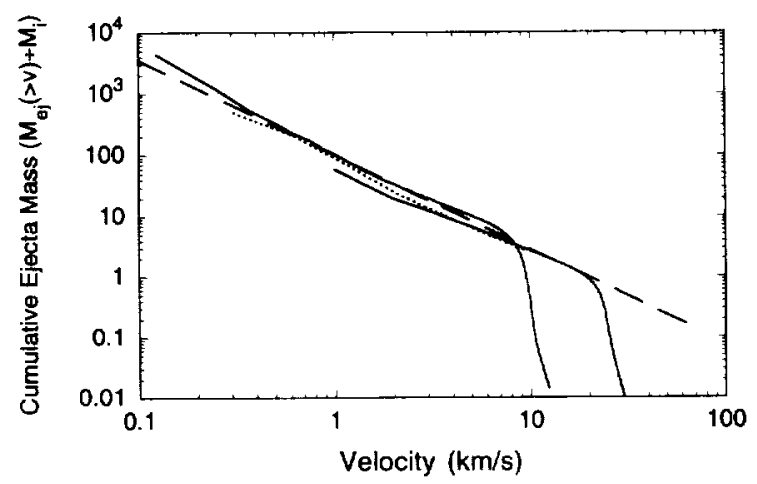

Figure 3. Cumulative mass-velocity distribution for two numerical models at times of $50 \mathrm{~s}$ (solid curve) and $100 \mathrm{~s}$ (dotted curve) after impact. The dashed line is a power law with exponent $\alpha=1.55$. expects. Immediately apparent from the figure is that the cumulative mass distribution does indeed approximate a power law for velocities below about $10 \mathrm{~km} / \mathrm{s}$. The power, $\alpha \approx 1.55$, is close to $\alpha$ for the idealized onedimensional impact. This is mostly coincidence, since a different numerical model (not shown), featuring a relatively shallow explosion, gives $\alpha=1.35$. The distribution truncates at a maximum velocity, call it $v_{\max }$, above which a much steeper distribution, $\alpha \sim 6$, obtains. Two specific cases are shown, one 4 times hotter than the other (constructed by setting the mass density in the wake 4 times lower). The hotter simulation gives higher maximum ejecta velocities. The cumulative kinetic energy in the ejecta plumes is quite large, about $35-40 \%$ of the total impact energy in both numerical models.

The explosion initially produces a small volume of very hot gas. This volume expands almost adiabatically, converting most of its initial thermal energy into kinetic energy. Because the fireball's radiating surface is relatively small, radiative losses are small. As the plume cools, pressure forces become unimportant and the velocity field becomes inertial and expansion homologous (shape-preserving), characterized by velocities increasing linearly with distance from the origin, such that

$$
\frac{v}{R}=\frac{v_{\max }}{R_{\max }}
$$

where $R$ is the radial distance from the origin, and $R_{\max }$ is the distance to the outer edge of the plume (defined by $v_{\max } t$ ). As these trajectories begin to bend under the influence of gravity a nearly purely ballistic velocity field results. The radial and vertical components of the ballistic velocity field are accurately given (rotation is ignored) by

$$
\begin{aligned}
& v_{r}=\frac{r}{t} \\
& v_{z}=\frac{z}{t}-\frac{1}{2} g t .
\end{aligned}
$$

We have verified this by directly superposing this simple velocity field on the velocity field calculated by detailed $2-D$ gas dynamical simulations (the 3 -D velocity field would be more complicated).

\section{Density}

For the toy plume we assume that the power law mass-velocity distribution applies after most of the thermal energy of the plume has been converted to kinetic energy. For simplicity, we also assume that the plume is axisymmetric and that it is initially ejected from a cone with opening angle $\theta^{\prime}$; i.e., ejection is isotropic for all angles $\theta<\theta^{\prime}$, where the opening angle $\theta^{\prime}$ is measured from the zenith. Our 2-D numerical models for candidate SL9 events typically explode with $\theta^{\prime} \approx 45^{\circ}$ [Zahnle and Mac Low, 1994a], although this is by no means an exact result. In general, the opening angle depends on the relative energy of the explosion. A very energetic explosion occurring very high in the atmosphere will 
have a wider opening angle than a low-energy explosion occurring deep in the atmosphere; the important dimensionless discriminant is $E_{i} / p_{0} H^{3}$, where $E_{\mathrm{i}}$ is the explosion energy, $p_{0}$ the ambient pressure, and $H$ the local scale height. Also, the numerical models appear to show that the opening angle is larger for high velocity material than for low-velocity material.

We can use the total mass and energy of the plume to obtain its density distribution as a function of ejection velocity (or, equivalently, as a function of position in the plume). Using (3) to relate $R$ and $v$, the cumulative mass $M(>v)$ in the plume is

$$
M(>v)=2 \pi\left(1-\cos \theta^{\prime}\right)\left(\frac{R_{\max }}{v_{\max }}\right)^{3} \int_{v}^{v_{\max }} \rho(v) v^{2} d v .
$$

It follows from (1) that $\rho(v) \propto v^{-3-\alpha}$. The cumulative kinetic energy of the plume is

$$
E(>v)=\pi\left(1-\cos \theta^{\prime}\right)\left(\frac{R_{\max }}{v_{\max }}\right)^{3} \int_{v}^{v_{\max }} \rho(v) v^{4} d v .
$$

Both energy and mass are conserved. If we assume that the plume is invested with a fraction $\eta$ of the total impact energy $\frac{1}{2} m_{i} v_{i}^{2}$, we obtain the density distribution

$$
\rho(v)=\frac{(2-\alpha) \eta m_{i}\left(v_{i} / v_{\max }\right)^{2}}{2 \pi\left(1-\cos \theta^{\prime}\right) R_{\max }^{3}}\left(\frac{v_{\max }}{v}\right)^{3+\alpha} .
$$

Equation (8) is valid for $v \leq v_{\max }$. In relating (8) to (6) we implicitly assume that $M\left(>v_{\max }\right)=0$. Note that, because $R_{\max }=v_{\max } t$, the density of the plume decays as $t^{-3}$, as expected for a homologous expansion. Our numerical results indicate that $0.3<\eta<0.45$; i.e., a substantial fraction of the impact energy is steered into the plume. This value may prove controversial, so we will treat $\eta$ as a parameter that others may set to 0.01 if they are so inclined.

The normalized form of $(1)$ is

$$
\begin{aligned}
M(>v) & =\frac{2-\alpha}{\alpha} \eta m_{i}\left(\frac{v_{i}}{v_{\max }}\right)^{2}\left(\frac{v_{\max }}{v}\right)^{\alpha} \\
& \approx 340 \eta m_{i}\left(\frac{1 \mathrm{~km} / \mathrm{s}}{v}\right)^{1.55} .
\end{aligned}
$$

Equation (9) has been evaluated for $\alpha=1.55$ and $v_{\max }=12 \mathrm{~km} / \mathrm{s}$, the latter consistent with a plume $3000 \mathrm{~km}$ high.

When the plume falls back onto the atmosphere, it liberates the vertical component of its kinetic energy in a reentry shock. There are actually two shocks, one driven into the Jovian atmosphere and the other in the falling plume. Both the plume and the atmosphere it strikes can get quite hot. Peak shock temperatures are highest in the more distantly thrown ejecta. The hot gas and embedded particulates cool radiatively.

The horizontal component of the velocity is preserved across the shock. Therefore the shocked plume continues to expand radially for a considerable time after it reenters the atmosphere. The plume expands horizontally until it has swept up its own mass in Jovian air; thus it travels about as far after it reenters the atmosphere as it does while in space. The characeristic crescent-shaped footprint of the plume was observed to have rotated through a larger angle than can be accounted for by Coriolis force acting only while the plume was in orbit [Hammel et al., 1995]. The additional rotation of the footprint was caused by the plume's continuing radial expansion for some 20-30 min after reentry.

We model the decline and fall of the plume by subdividing the early plume three dimensionally $(r, \theta, \phi)$ into a vast number of mass elements. Each mass element is launched on its own ballistic trajectory. We then count up where and when the elements reenter the atmosphere. This approach is computationally intensive, and hence well-suited to the expensive and astonishingly powerful machines that would otherwise sit idle on our desktops. The mass of a finite element of the plume with velocity $v$, altitude $\theta$, and azimuth $\phi$ is

$$
\delta m=\delta \phi \sin \theta \delta \theta \rho(v) v^{2} \delta v\left(\frac{R_{\max }}{v_{\max }}\right)^{3} .
$$

The mass element reenters the atmosphere a horizontal distance

$$
r(v, \theta)=v^{2} \sin 2 \theta / g
$$

from the origin at time

$$
t(v, \theta)=2 v \cos \theta / g
$$

as determined by conventional ballistics in the flat Jupiter approximation.

\section{Pressure}

The pressure level of the reentry shock, if strong, is

$$
p^{\prime}=\frac{\gamma+1}{2} \rho v_{z}^{2} \text {. }
$$

This can be evaluated directly to give $p^{\prime}$ as a function of position and time, but this is not as useful or as accurate a measure of "the" shock pressure as one might hope. To first approximation, the greatest mass flux of material to arrive at any point is the first material to get there. This is rigorously true for plumes with opening angles $\theta^{\prime} \leq 45^{\circ}$, for which the first material to reach a given point is ejected with the lowest total velocity of any material to reach that point, and hence it is the densest gas to reach that point. Thereafter the mass flux declines monotonically and the shock rises to progressively lower pressures. Meanwhile the heated atmosphere both cools radiatively and also rises to seek a new scale height. The accumulated mass fallen at a given place (multiplied by $g$ ) provides a measure of the pressure level of the contact discontinuity between the plume and the Jovian atmosphere, and we suggest that this is probably a good measure of the reentry shock.

An illustrative analytic approximation to the pressure at the reentry shock can be obtained by replacing $v^{2}$ in (9) by $r g$, which gives 


$$
\begin{aligned}
p(r) & =\frac{d M / d r}{2 \pi r} g \\
& \approx \frac{(2-\alpha) \eta m_{i}}{4 \pi}\left(\frac{v_{i}}{v_{\max }}\right)^{2} \frac{v_{\max }^{\alpha} g^{1-\alpha / 2}}{r^{2+\alpha / 2}}
\end{aligned}
$$

When evaluated for $\alpha=1.55$ and $v_{\max }=12 \mathrm{~km} / \mathrm{s}$, this becomes

$$
p(r) \approx 90 \eta\left(\frac{m_{i}}{10^{14} \mathrm{~g}}\right)\left(\frac{1000 \mathrm{~km}}{r}\right)^{2.8} \mu \text { bars. }
$$

Shock pressure is directly proportional to the mass of the impactor. Pressures at large distances are quite low. At $r=2000 \mathrm{~km}$, a typical shock pressure produced by a $10^{14} \mathrm{~g}$ impact with $\eta=0.4$ would be $5 \mu$ bars.

In Figure 4 we compare the pressure level given by (15) (faded solid curve) to results obtained directly from our fiducial numerical model (diamonds). This is the same numerical model by which we calibrated (9), so good agreement might be expected; nevertheless, the agreement is gratifyingly good. The diamonds indicate where tracer particles swept up in the plume have gotten to after $15 \mathrm{~min}$. The lowermost diamonds trace the location of the boundary between Jupiter and the ejecta.

\section{Temperature}

The ordinary Rankine-Hugoniot relations for a strong shock require that the infalling material reach a temperature of

$$
T^{\prime} \geq \frac{\gamma-1}{2} \frac{m}{k} v^{2}=1500 \mathrm{~K}\left(\frac{\mu}{2.5}\right)\left(\frac{v}{5 \mathrm{~km} / \mathrm{s}}\right)^{2},
$$

where $m$ is the mean molecular mass. The origin of this equation can also be seen as a balance of the initial kinetic energy $\frac{1}{2} v_{z}^{2}$ with the thermal energy $c_{v} T$ of the halted gas (for a weak shock, temperatures are higher than given by (16)).

The temperature in (16) is the temperature of the gas immediately after it passes through the shock. It

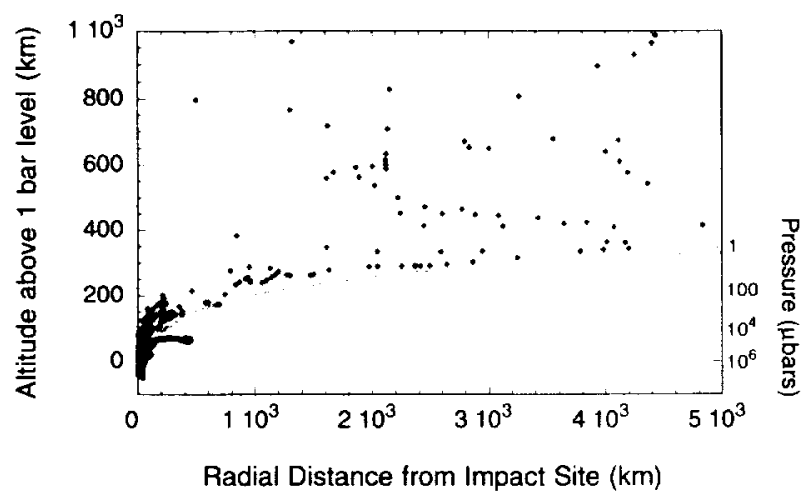

Figure 4. The altitude and pressure of the reentry shock for a $10^{27}$ erg SL9 impact, 15 min after impact. The solid curve shows the location of the reentry shock as approximated by (15). The diamonds show tracer particles that were swept up by the plume. The lowest diamonds trace the boundary between Jupiter and the fallen plume. can be high. Temperatures would be especially high in material that originated from the comet because the mean molecular weight of the vaporized comet is relatively large, probably of the order of $10<\mu<20$. The detection of hot $(>2000 \mathrm{~K})$ carbon monoxide emission at $2.3 \mu \mathrm{m}$ (R. Knacke, personal communication, 1994); [Meadows et al., 1994] offers direct evidence that former cometary material became very hot on reentering the atmosphere.

The effective radiating temperature, between 500 and $1000 \mathrm{~K}$ [Nicholson et al., 1995b], was much lower than implied by (16). Evidently radiative cooling by the shocked material was efficient. HST images clearly revealed that the ejecta blankets were dusty [Hammel et al., 1995]. The dust is an obvious opacity source for radiative cooling.

Perhaps the simplest expression for the radiating temperature of a dusty layer heated by falling ejecta is to assume an instantaneous energy balance between the kinetic energy of falling matter and radiative cooling, buffered by the heat capacity of the falling matter [Zahnle, 1990; Melosh et al., 1990]:

$$
\dot{e}=\frac{1}{\gamma-1} \frac{k T}{m} \dot{m}+2 \sigma T^{4}\left(1-e^{-2 \tau}\right) .
$$

The factor $2 \sigma T^{4}$ assumes a grey radiator that emits both up and down. The factor $1-e^{-2 \tau}$ allows for the optical depth $\tau$ of the radiating layer; the factor of 2 gives the right limit as $\tau \rightarrow 0$. We solve (17) for the temperature $T$ by Newton's method.

\section{Opacity}

Radiative cooling depends on the presence of an opacity source, presumably particulates either in the plume or generated when the plume strikes the atmosphere. The latter requires a sufficiently strong shock. Zahnle et al. [1995] show that reentry velocities must exceed $4-5 \mathrm{~km} / \mathrm{s}$ for shock heating to be strong enough to generate carbonaceous dust from Jovian air. This can explain the relative transparency of the inner parts of the ejecta blanket as observed with the HST [Hammel et al., 1995], and it is probably required to explain the late onset of infrared radiation as observed by the Galileo spacecraft [Carlson et al., 1995a,b]. The observed particulates are apparently rather small. Nicholson et al. [1995b] suggest an effective optical depth of about 0.022 at $\lambda_{0}=4.5 \mu \mathrm{m}$, with a $\lambda^{-1}$ dependence. Molecular opacity might also contribute. Here we will use a simple grey (wavelength-independent) opacity.

We assume that $\tau$ is proportional to the total integrated mass of material falling at radius $r$,

$$
\tau(r, t)=\kappa \int_{0}^{t} \dot{m} d t
$$

i.e., the opacity sources are embedded uniformly in the shocked air, and all the dust that falls at any point is available to contribute to radiative cooling. This approximation ignores the vertical structure of the reentry shock (late arriving material shocks at higher altitudes) and radial gradients in the plume's composition (the 
more distantly thrown material may be comet-rich, and hence carry more grains).

Figure 5 shows effective radiating temperatures at 5 , 10 , and $15 \mathrm{~min}$, as a function of distance from the impact site, calculated with a constant $\kappa=100 \mathrm{~cm}^{2} / \mathrm{g}$ (solid curves). This is the plume used to generate the light curves shown in Figures 6 and 7 . The temperature field forms warm rings (seen nicely at $10 \mathrm{~min}$ ). The inner regions are cooler because the optical depth is relatively high (from early-arriving ejecta) and because temperature is calculated by the instantaneous energy balance between $\dot{e}$ and radiative cooling. If either of these assumptions is relaxed the expected interior temperature warms. To illustrate this, the dotted temperatures on Figure 5 were generated from the same model, modified to limit optical depth to material arriving in the previous $90 \mathrm{~s}$ :

$$
\tau(r, t)=\kappa \int_{t-90}^{t} \dot{m} d t .
$$

The temperatures that result are much more nearly constant, both in radius and time.

\section{Geometry}

The impact occurs at an angle $\theta_{0}$ over the horizon. A given element of the plume reenters the atmosphere a time $t$ later, at a distance $r$ from the impact site, and at an azimuth $\phi$ (measured from north). During this time the impact site rotates into view at a rate of $0.01^{\circ} \mathrm{s}^{-1}$. The projected surface area of the plume's footprint as viewed from Earth is proportional to the cosine of the angle $\psi$ between the surface normal and the line to Earth

$$
\cos \psi \approx \sin \left(\frac{r \sin \phi}{R_{J} \cos l_{i}}-\theta_{0}+1.77 \times 10^{-4} t\right)>0,
$$

where $l_{i} \approx 45^{\circ}$ denotes the latitude of the impact, and $R_{J}$ refers to Jupiter's radius. Where $\cos \psi<0$ the surface element is on the far side of the planet. The

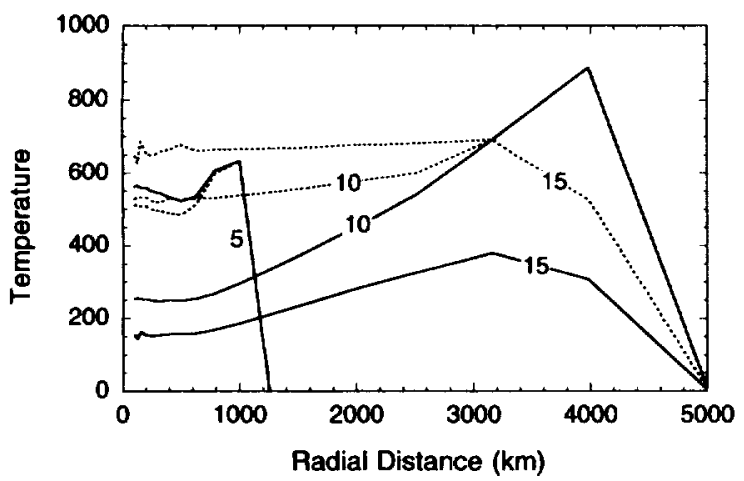

Figure 5. Effective radiating temperatures at 5, 10, and $15 \mathrm{~min}$ produced by the toy plume with constant opacity $\kappa=100 \mathrm{~cm}^{2} / \mathrm{g}$. The solid curves use (18), to which all the particles contribute to optical depth. This is our nominal standard model. The dotted curves use (19), to which only the most recently arriving particles contribute to the effective optical depth.

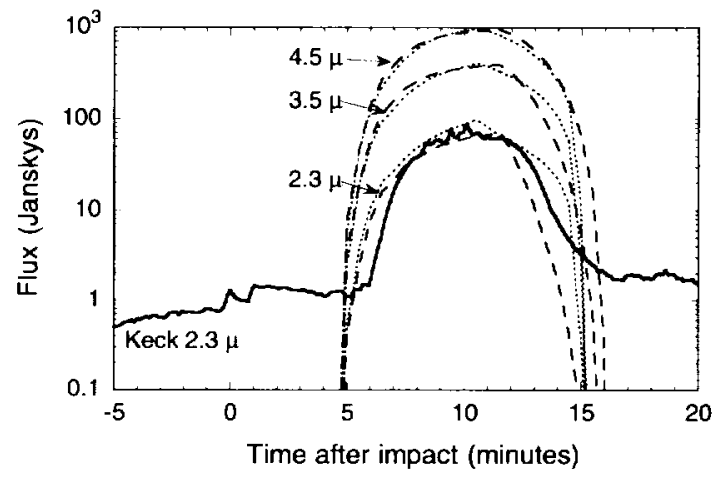

Figure 6. Light curves at selected infrared wavelengths generated by the toy plume model, as seen from Earth. The Keck light curve [Graham et al., 1995] at $2.3 \mu \mathrm{m}$ is shown for comparison (solid curve). The dashed curves (standard model) uses (18) for $\tau(r, t)$; the impactor is $500 \mathrm{~m}$ diameter and $0.5 \mathrm{~g} / \mathrm{cm}^{3}$. The dotted curves use (19) for $\tau(r, t)$ and a $450 \mathrm{~m}$ impactor

flux at Earth is

$$
F_{\nu}=\frac{B_{\nu}}{A^{2}} \sum \cos \psi d \sigma\left(1-\exp \left(-\tau_{\nu} \sec \psi\right)\right),
$$

where $B_{\nu}$ is the Planck function, $A$ the Earth-Jupiter distance, and $d \sigma$ a surface element on Jupiter.

We have neglected azimuthal asymmetry in (21); this will be important to a complete model. We have also neglected the altitude of the radiating layer. Altitude can strongly affect visibility. When we attempt to account for this, by using the numerical equivalent of (14) and (15) for pressure, visibility from Earth generally improves. The result is a brighter, earlier flash. For the present we choose to omit this parameter. In a future study, one that includes a nonaxisymetric 3-D plume, we will consider the role of altitude more fully.

\section{Light}

Figure 6 shows calculated light curves at $2.3,3.5$, and $4.5 \mu \mathrm{m}$, as seen from Earth, generated by the toy plume models (dashed, dotted lines). The dashed curves use (18) to prescribe optical depth. The Keck light curve for fragment R [Graham et al., 1995] at $2.3 \mu \mathrm{m}$ is shown for comparison. The match at $3.5 \mu \mathrm{m}$ and $4.5 \mu \mathrm{m}$ is comparably good (or bad, depending on one's taste in these things; see Nicholson et al. [1995a]. This model uses a $500 \mathrm{~m}$ diameter impactor of density $0.5 \mathrm{~g} / \mathrm{cm}^{3}$, $\eta=0.5, v_{\max }=12 \mathrm{~km} / \mathrm{s}$, and an opening angle $\theta^{\prime}=$ $45^{\circ}$. The total impact energy is $6 \times 10^{26}$ ergs. The value of $v_{\max }, 12 \mathrm{~km} / \mathrm{s}$, implies a plume that reaches $3000 \mathrm{~km}$ high. The value of $v_{\max }$ is set by the duration of peak light, and hence is not very negotiable.

We obtain a good match to the relative fluxes at 2.3 , 3.5 , and $4.5 \mu \mathrm{m}$ using a constant opacity of $\kappa=100$ $\mathrm{cm}^{2} / \mathrm{g}$, which is fairly high. If we assume particles of radius $0.25 \mu \mathrm{m}$, density of $1 \mathrm{~g} / \mathrm{cm}^{3}$, and assume that opacity is equal to the sum of their geometric cross sections, the particle density in the ejecta plume would be $0.004 \mathrm{~g} / \mathrm{g}$. If instead we assume that their effective 


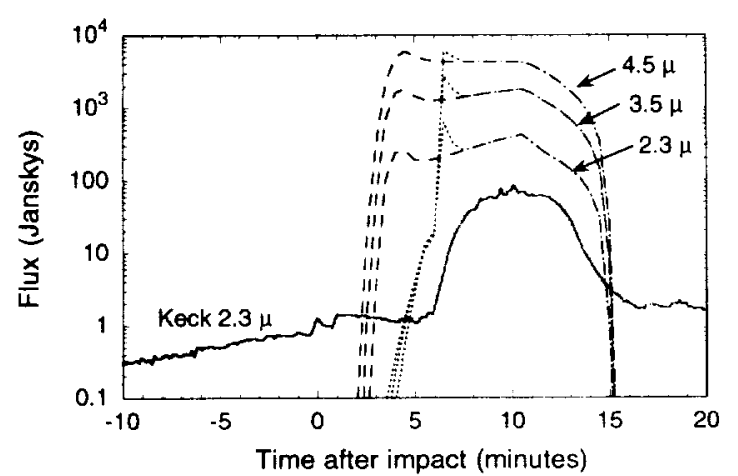

Figure 7. Light curves at selected infrared wavelengths generated as seen from directly above the impact site. This view more closely approximates Galileo's. The dashed curves are from our standard model. The onset is too early. The dotted curves restrict dust opacity to air experiencing shock velocities exceeding $4.5 \mathrm{~km} / \mathrm{s}$. This approximates the minimum shock heating required to generate carbonaceous particulates from Jovian air [Zahnle et al., 1995]. The "spikes" in the light curves are probably artificial, caused by relatively high temperatures when optical depth is very low.

cross sections are proportional to $\lambda^{-1}$, the required particle density in the plume rises to $0.04-0.08 \mathrm{~g} / \mathrm{g}$. Such a high density would require that a fair fraction of the plume is of cometary origin. According to Pollack et al. [1994], the Rosseland mean opacity of a solar composition mix of gas and dust, in which $60 \%$ of the carbon is in organic grains, is about $\kappa=7 \mathrm{~cm}^{2} / \mathrm{g}$; for shocked Jovian air the corresponding opacity would be $\kappa \approx 20-30$ $\mathrm{cm}^{2} / \mathrm{g}$. Again, to raise the opacity to $\kappa \approx 100 \mathrm{~cm}^{2} / \mathrm{g}$ requires a significant admixture of comet.

The calculated light curves using (18) for $\tau$ (dashed lines) decay too abruptly, especially at $2.3 \mu \mathrm{m}$. This occurs in our standard model because (1) the temperature drops rapidly once most of the mass of the plume has reentered the atmosphere, caused by the optical depth (18) remaining high while $\dot{e}$ declines, and (2) the plume is artificially truncated at $v<v_{\max }$, which effectively terminates the event at $t=2 v_{\max } / g$. There are several effects that could act to smooth the light curve. The continued radial spreading of the ejecta pattern after reentry, alluded to above, is one effect. A second is finite heat capacity. We have assumed an instantaneous energy balance. Allowing for history would moderate the temperature decline. A third, also discussed above, is that the optical depth of the earliest arriving ejecta may not be relevant to the late arriving ejecta. On the other hand, it is interesting to note that the general form of the computed light curve at $2.3 \mu \mathrm{m}$ is very similar to that of the bright $\mathrm{K}$ event [Watanabe et al., 1995]. It would appear that the smaller, optically thinner events produced smoother light curves, while the larger, optically thicker events were more asymmetric.

The dotted curves on Figure 6 show how the light curves change when (19) is used to prescribe $\tau$. The result is that the light curves at $2.3,3.5$, and $4.5 \mu \mathrm{m}$ are smoother and more nearly parallel, as observed [Nicholson et al., 1995a]. To match the observed fluxes this model uses a $450 \mathrm{~m}$ rather than a $500 \mathrm{~m}$ impactor. In this model $v_{\max }=11 \mathrm{~km} / \mathrm{s}$ (the best fit is with $v_{\max }=10.8 \mathrm{~km} / \mathrm{s}$ ).

Figure 7 shows the same event ( $450 \mathrm{~m}$ object) as seen from directly above the impact site. These light curves approximate the view from Galileo. Because the radiating layer is mostly optically thin, the fluxes seen from Galileo are not likely to be much smaller. The event is brighter when seen centered on Jupiter's disk, and it starts earlier. The predicted early rise (dashed curve) was not seen by Galileo for the $\mathrm{R}$ and $\mathrm{G}$ impacts [Carlson et al, 1995b]. The delayed onset of radiation from the reentry of the plume is not plausibly explained by an absence of low-velocity ejecta. A better explanation, and one that receives direct visible support from the spectacular HST photos, is that low-velocity ejecta were largely transparent. Low-velocity ejecta are probably relatively comet-free, hence to become opaque the air must generate its own opacity. Zahnle et al. [1995] show that if reentry velocities exceed $4.5 \mathrm{~km} / \mathrm{s}$ the shock is strong enough to generate carbonaceous dust, possibly soot, from Jovian air. The dotted curves show the consequences on the Galileo light curve of restricting dust formation to air shocked at $v_{z}>4.5 \mathrm{~km} / \mathrm{s}$. The fast rise at $6 \mathrm{~min}$ is a probably artificial consequence of turning opacity on abruptly at $4.5 \mathrm{~km} / \mathrm{s}$; when the opacity is first turned on, the temperature is too high.

Taken at face value, the toy plume model implies that the $R$ event was produced by the impact of a 450 to 500 m diameter body of mass $2 \times 10^{13}$ to $3 \times 10^{13} \mathrm{~g}$. By extension the largest plumes would be created by $1 \times 10^{14}$ to $2 \times 10^{14} \mathrm{~g}$ objects. These sizes are consistent with the tidal-breakup models of Asphaug and Benz [1994], and Solem [1994]. However, energy estimates made by the light of the plume are somewhat sensitive to opacity and viewing geometry. The view from Galileo is less sensitive to geometrical details and hence should provide a better observational constraint on the actual energy of the plume.

\section{Appendix: Initial Conditions to Numerical Models}

The initial conditions of the explosions assume the bolide wake can be represented by a moving line charge with an energy deposition rate described by the analytic theory of Zahnle and Mac Low [1994a]. There we made the assumption that the impactor expands quasi-statically due to the pressure difference between its front and sides to derive an approximate analytic expression for its downward velocity,

$$
v=v_{i} \exp \left(\frac{-\pi C_{D}^{2} \rho(z)^{2} H^{3} \sec ^{3} \theta}{2 m_{i} \rho_{i}}\right),
$$

where the impactor has an initial downward velocity $v_{i}$ at an angle to the horizontal of $\theta$, a density of $\rho_{i}$, and a coefficient of drag $C_{D}$, which has values of 1.0 for a 
sphere and 1.7 for a cylinder. The atmosphere has a density profile $\rho$ and a scale height $H$.

We need to make one further approximation in order to derive an analytic solution for the energy release profile. We approximate the density profile as a simple exponential, $\rho=\rho_{0} \exp (-z / H)$. In the simulation we actually use a more realistic atmosphere with variable scale height [see Zahnle and Mac Low, 1994a], so we take $H$ to be the value near the point of maximum energy release. We also add a term to account for high altitude radiative ablation to get

$$
\frac{1}{\rho} \frac{d E}{d z}=\frac{\pi v^{2} \rho C_{D}^{2} H^{2} \sec ^{3} \theta}{\rho_{i}}+\frac{1}{2} C_{D} \pi r_{i}^{2} v^{2} \sec \theta,
$$

where the initial radius of the impactor is $r_{i}$.

The radius of the wake is a parabola in altitude $z$,

$$
R(z) \approx \sqrt{r_{i}^{2}+\left(\frac{4}{\pi} \frac{(\gamma+1)^{2}(\gamma-1)}{3 \gamma+1}\right)^{1 / 2} \frac{1}{\rho} \frac{d E}{d z} \frac{z-z_{0}}{v_{i}}}
$$

where $z_{0}$ is the airburst altitude [Zahnle and Mac Low, $1994 \mathrm{a}$; equation 5]; $r_{i}$ corresponds to the radius of the impactor, and $v_{i}$ to its velocity. We place the bottom of the wake at an altitude $z^{\prime}$ slightly below $z_{0}$, at

$$
z^{\prime}=z_{0}-\left(2 C_{D} \rho / \rho_{i}\right)^{1 / 2} H \sec \theta-r_{i} .
$$

The wake is assumed to be well-mixed so that energy and mass are uniformly distributed across the wake. Its velocity is also neglected, since a moving line charge has no momentum. The energy density in the wake is then

$$
e^{\prime}(z)=e(z)+\frac{1}{\pi R^{2}(z)} \frac{d E}{d z}
$$

and the mass density in the wake is

$$
\rho^{\prime}=\rho+\frac{1}{\pi R^{2}(z)} \frac{d m}{d z} .
$$

The assumption of a uniform wake is not a very good one. In reality most of the mass and energy of the wake are concentrated at the surface of the paraboloid, while the interior is mostly evacuated. However, the numerical model requires several grid elements to resolve a shock. In practice more realistic initial conditions requires impractically high resolution in the wake.

The models used to generate the light curves in Figure 2 take $z_{0}=-80 \mathrm{~km}\left(10^{28} \mathrm{ergs}, r_{i}=0.5 \mathrm{~km}\right)$ and $z_{0}=-25 \mathrm{~km}\left(10^{27} \mathrm{ergs}, r_{i}=0.236 \mathrm{~km}\right)$; both use $\gamma=1.2$. The models used to generate the cumulative mass-velocity curves in Figure 3 take $z_{0}=-25 \mathrm{~km}$, $r_{i}=0.297 \mathrm{~km}, m_{i}=1.1 \times 10^{14} \mathrm{~g}, E_{i}=2 \times 10^{27} \mathrm{ergs}$, and $\gamma=1.4$; the hotter model (larger $v_{\max }$ ) was generated by reducing the mass density in the wake by a factor of 6.8 .

Acknowledgments. We thank James Graham, Imke de Pater, Phil Nicholson, and Torrence Johnson for the endless conversation and timely access to their ever-evolving data, and for explaining the facts surrounding the first precursor to K.Z. We also thank the Hubble team, in particular Heidi Hammel, John Clarke, Walt Harris, Keith Noll, and Andy Ingersoll, for their part in the conversation. M.-M. M. L. was supported by NSF grant AST93-22509 from the NSF Aeronomy Program. K.Z. was supported by the NASA Exobiology Program. They suspect but do not know.

\section{References}

Ahrens, T., T. Takata, J. D. O'Keefe, and G. Orton, Impact of Comet Shoemaker-Levy 9 on Jupiter, Geophys. Res. Lett., 21, 1087-1090, 1994.

Asphaug, E. and W. Benz, Density of comet ShoemakerLevy 9 deduced by modelling breakup of the parent "rubble pile', Nature, 370, 120-124, 1994.

Boslough, M., D. Crawford, A. Robinson, and T. Trucano, Mass and penetration depth of Shoemaker-Levy 9 fragments from time-resolved photometry, Geophys. Res. Lett., 21, 1555-1558, 1994.

Carlson, R., et al., Galileo NIMS observations of the impact of comet Shoemaker-Levy 9 on Jupiter, (abstract), EOS Trans. $A G U, 75$, Fall Meet. Suppl., 401, 1994.

Carlson, R., et al., Galileo infrared observations of the Shoemaker-Levy 9 G impact fireball: a preliminary report, Geophys. Res. Lett., in press, 1995.

Carlson, R., et al., Some timing and spectral aspects of the $G$ and $R$ collision events as observed by the Galileo near-infrared mapping spectrometer, Proceedings: European Shoemaker-Levy 9 Conference, 1995b.

Chapman, C., W. Merline, K. Klaasen, T. Johnson, C. Heffernan, M. Belton, A. Ingersoll, and the Galileo imaging team, Preliminary results of Galileo direct imaging of SL9 impacts, Geophys. Res. Lett., in press, 1995a.

Chevalier, R. and C. Sarazin, Explosions of infalling comets in Jupiter's atmosphere, Astrophys. J., 429, 863-875, 1994.

Crawford D., M. Boslough, T. Trucano, and A. Robinson, Numerical simulations of fireball growth and ejecta distribution during Shoemaker-Levy 9 impact on Jupiter, (abstract), EOS Trans. $A G U$, 75, Fall Meet. Suppl., 404, 1994.

Graham, J., I. de Pater, J. G. Jernigan, M. Liu, and M. Brown, W. M. Keck telescope observations of the comet P/Shoemaker-Levy 9 fragment R Jupiter collision, $S c i$ ence, 267, 1320-1323, 1995.

Hammel, H., et al., Hubble Space Telescope imaging of Jupiter: atmospheric phenomena created by the impact of comet Shoemaker-Levy 9, Science, 267, 1288-1296, 1995.

Housen, K., R. Schmidt, and K. Holsapple, Crater ejecta scaling laws: Fundamental forms based on dimensional analysis, $J$. Geophys. Res., 88, 2485-2499, 1983.

Mac Low, M.-M. and K. Zahnle, Explosion of Comet Shoemaker-Levy 9 on entry into the Jovian atmosphere, $\mathrm{As}$ trophys. J. Lett., 434, L33-L36, 1994.

Martin, T., L. Tamppari, G. Orton, I. Claypool, and L. Travis, Galileo PPR observations of Shoemaker-Levy 9, Science, 267, 1995.

Meadows, V., D. Crisp, G. Orton, T. Brooke, and J. Spencer, AAT observations of Shoemaker-Levy 9 collisions with Jupiter, poster presented at the $26^{\text {th }}$ Ann. Mtg., Div. Planet. Sci., Bethesda MD, Oct. 31-Nov. 4, 1994.

Melosh, H. J., N. Schneider, K. Zahnle, and D. Latham, Ignition of global wildfires at the Cretaceous/Tertiary boundary, Nature, 343, 251254, 1990.

Nicholson, P., et al., Palomar observations of the impact of the $\mathrm{R}$ fragment of comet $\mathrm{P} /$ Shomeaker-Levy 9: I. Light curves, Geophys. Res. Lett., in press, 1995a. 
Nicholson, P., et al., Palomar observations of the impact of the $\mathbf{R}$ fragment of comet $\mathrm{P} /$ Shomeaker-Levy 9: II. Spectra, Geophys. Res. Lett., in press, 1995b.

Pollack, J. B., D. Hollenbach, S. Beckwith, D. Simonelli, T. Roush, and W. Fong, Composition and radiative properties of grains in molecular clouds and accretion disks, Astrophys. J., 412, 615-639, 1994.

Schmidt, $R$. and $K$. Housen, Some recent advances in the scaling of impact and explosion cratering, Int. J. Imp. Eng., 5, 543-560, 1987.

Solem, J. C., Density and size of comet Shoemaker-Levy 9 deduced from a tidal breakup model, Nature, 370, 349$351,1994$.

Watanabe, J., T. Yamashita, T. Hasegawa, S. Takeuchi, M. Abe, Y. Hirota, E. Nishihara, S. Okumura, and A. Mori, Near-IR observation of cometary impacts to Jupiter: Brightness variation of the impact plume of fragment K, Publ. Astron. Soc. Jpn., 47, L21-L24, 1995.

Zahnle, K., Atmospheric chemistry by large impacts, Global Catastrophes in Earth History, edited by V. Sharpton and P. Ward, Geol. Soc. Am. Spec. Pap. 247, 271288, 1990.
Zahnle, K. and M.-M. Mac Low, The collision of Jupiter and Comet Shoemaker-Levy 9, Icarus, 108, 1-17, 1994 a.

Zahnle, K. and M.-M. Mac Low, The end of Comet Shocmaker-Levy 9, (abstract), EOS Trans. AGU, 75, 402, $1994 \mathrm{~b}$.

Zahnle, K., M.-M. Mac Low, K. Lodders, and B. Fegley, Sulfur chemistry in the wake of Shoemaker-Levy 9, Geophys. Res. Lett., in press, 1995.

Zel'dovich, Ia. B. and Yu. P. Raizer, Physics of Shock Waves and High Temperature Hydrodynamic Phenomena, vol. II, Academic, San Diego; 1967.

K. Zahnle, NASA Ames Research Center, MS 245-3, Molfett Field, CA 94035-1000.

M.-M. Mac Low, Department of Astronomy and Astrophysics, 5640 S. Ellis Ave., University of Chicago, Chicago IL 60637 .

(Received February 27, 1995; revised May 24, 1995; accepted May 24, 1995.) 